\title{
Relação entre aleitamento e desenvolvimento do sistema estomatognático: revisão
}

\section{sistemática}

\author{
Relationship between breastfeeding and development of the stomatognathic system: systematic \\ review
}

Relación entre lactancia y desarrollo del sistema estomatognático: revisión sistemática

Recebido: 30/08/2021 | Revisado: 04/09/2021 | Aceito: 06/09/2021 | Publicado: 07/09/2021

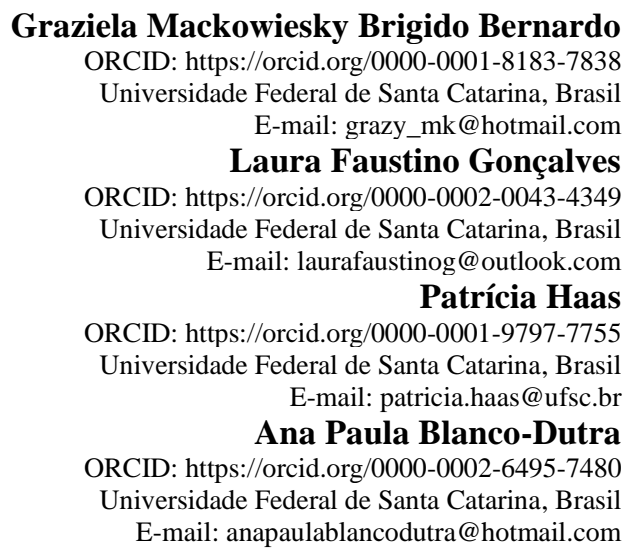

\begin{abstract}
Resumo
Introdução: A amamentação promove benefícios, tanto para o recém nascido, quanto para a mãe. Apesar da importância do aleitamento materno, há dificuldades peculiares para a mãe e o bebê no período de internação, podendo levar ao desmame precoce. Objetivo: Verificar a relação entre aleitamento e os reflexos na motricidade orofacial. Metodologia: A busca por artigos científicos foi conduzida por dois pesquisadores independentes nas bases de dados MEDLINE (Pubmed), LILACS, SciELO, SCOPUS, WEB OF SCIENCE e BIREME, sem restrição de idioma e localização, durante o período de 2010 a 2021. Para complementar e evitar viés de risco foi realizada uma busca por literatura cinza no Google Scholar. A revisão sistemática foi conduzida conforme as recomendações do Preferred Reporting Items for Systematic Reviews and Meta-Analyses (PRISMA). Foram inclusos na pesquisa estudos que obtiveram pontuação $\geq$ a 12 pontos segundo o protocolo para pontuação qualitativa proposto por Pithon et al. (2015). Resultados: A duração do aleitamento materno associou-se positivamente à qualidade da função mastigatória. Os estudos mostraram que a amamentação tem um impacto positivo na função mastigatória e de sucção. Conclusão: Ao relacionar os achados desta pesquisa com o desmame precoce e seus reflexos no sistema estomatognático evidencia-se que o aleitamento materno natural oferece ao bebê um adequado desenvolvimento ósseo e muscular, garantindo, assim, o perfeito funcionamento das estruturas orais e possibilitando saúde geral devido às interrelações existentes entre o sistema estomatognático e os demais órgãos e funções.
\end{abstract}

Palavras-chave: Recém-nascido; Aleitamento materno; Fonoaudiologia; Desmame.

\begin{abstract}
Introduction: Breastfeeding promotes benefits for both the newborn and the mother. Despite the importance of breastfeeding, there are peculiar difficulties for the mother and the baby during the hospitalization period, which can lead to early weaning. Objective: To verify the relationship between breastfeeding and reflexes on orofacial motricity. Methodology: The search for scientific articles was conducted by two independent researchers in the MEDLINE (Pubmed), LILACS, SciELO, SCOPUS, WEB OF SCIENCE and BIREME databases, with no restriction on language and location, during the period from 2010 to 2021. For Complementary and avoiding risk bias, a search for gray literature in Google Scholar was performed. The systematic review was conducted according to the recommendations of the Preferred Reporting Items for Systematic Reviews and Meta-Analyses (PRISMA). Studies that obtained a score $\geq$ to 12 points according to the protocol for qualitative scoring proposed by Pithon et al. (2015). Results: The duration of breastfeeding was positively associated with the quality of masticatory function. Studies have shown that breastfeeding has a positive impact on chewing and sucking function. Conclusion: By relating the findings of this research with early weaning and its effects on the stomatognathic system, it is evident that natural breastfeeding offers
\end{abstract}


the baby adequate bone and muscle development, thus ensuring the perfect functioning of oral structures and enabling general health due to the interrelationships between the stomatognathic system and the other organs and functions.

Keywords: Newborn; Breastfeeding; Speech therapy; Weaning.

\section{Resumen}

Introducción: La lactancia materna promueve beneficios tanto para el recién nacido como para la madre. A pesar de la importancia de la lactancia materna, existen dificultades peculiares para la madre y el bebé durante el período de hospitalización, que pueden llevar a un destete precoz. Objetivo: Verificar la relación entre la lactancia materna y los reflejos sobre la motricidad orofacial. Metodología: La búsqueda de artículos científicos fue realizada por dos investigadores independientes en las bases de datos MEDLINE (Pubmed), LILACS, SciELO, SCOPUS, WEB OF SCIENCE y BIREME, sin restricción de idioma y ubicación, durante el período de 2010 a 2021. Para De forma complementaria y evitando el sesgo de riesgo, se realizó una búsqueda de literatura gris en Google Scholar. La revisión sistemática se llevó a cabo de acuerdo con las recomendaciones de los ítems de informes preferidos para revisiones sistemáticas y metaanálisis (PRISMA). Los estudios que obtuvieron puntuaciones $\geq$ a 12 puntos según el protocolo de puntuación cualitativa propuesto por Pithon et al. (2015). Resultados: La duración de la lactancia materna se asoció positivamente con la calidad de la función masticatoria. Los estudios han demostrado que la lactancia materna tiene un impacto positivo en la función de masticar y succionar. Conclusión: Al relacionar los hallazgos de esta investigación con el destete precoz y sus efectos sobre el sistema estomatognático, se evidencia que la lactancia natural ofrece al bebé un adecuado desarrollo óseo y muscular, asegurando así el perfecto funcionamiento de las estructuras bucales y posibilitando la salud general debido las interrelaciones entre el sistema estomatognático y los demás órganos y funciones.

Palabras clave: Recién nacido; Amamantamiento; Terapia del lenguaje; Destete.

\section{Introdução}

$\mathrm{O}$ aleitamento materno (AM) tem sido alvo de pesquisas devido à crescente evidência da importância dessa prática, sendo considerada como promotora de saúde física e psicológica da criança e da mulher que amamenta, além de prevenir mortes infantis (Bueno, Gomez, 2013).

Segundo a Organização das Nações Unidas - OMS (2001), é recomendada a amamentação materna exclusiva (AME) por 4-6 meses e complementada por 2 anos ou mais, pois não há vantagens de introduzir alimentos complementares antes dos seis meses, podendo acarretar prejuízos para o desenvolvimento do bebê.

A amamentação promove benefícios, tanto para o recém nascido (RN), quanto para a mãe. Ela fornece toda a energia e nutrientes que o recém nascido necessita. Além de conter linfócitos e imunoglobulinas que auxiliam no sistema imunológico do bebê, protegendo contra doenças crônicas e infecciosas, ainda promove o desenvolvimento sensório-motor e cognitivo da criança (Coelho et al., 2019). Da mesma forma, a mãe pode ser beneficiada no processo de amamentação, visto que, o mesmo ajuda na recuperação pós-parto, favorecendo a estimulação da oxitocina, auxiliando na prevenção de uma nova gestação, além de contribuir na redução de risco de câncer de mamas, útero e ovários (Brasil, 2014). Além de trazer todos os benefícios citados anteriormente, a amamentação promove o harmônico desenvolvimento craniofacial do bebê pelos movimentos adequados da musculatura orofacial.

Diante disso, é importante observar algumas habilidades para uma amamentação adequada, à sucção, posição dos lábios durante a amamentação no seio, apreensão do mamilo, entre outros aspectos (Delgado, Halpern, 2005). A importância da sucção durante a amamentação promove o desenvolvimento adequado dos órgãos fonoarticulatórios - OFAs (lábios, língua, mandíbula, maxila, bochechas, palato mole, palato duro, soalho da boca, musculatura oral e arcadas dentárias) quanto à mobilidade, força e postura, além de auxiliar o desenvolvimento das funções de respiração, mastigação, deglutição e articulação dos sons da fala (Neiva et al., 2003).

Apesar da importância do AM estar estabelecida na literatura, há dificuldades peculiares para a mãe, para o bebê e para os profissionais da saúde no período de internação (Scheeren et al., 2012). Essas dificuldades podem levar ao desmame precoce. A OMS e o Fundo das Nações Unidas para a Infância (UNICEF), em 1980, definiram o desmame como um processo pelo qual são introduzidos gradualmente na dieta da criança outros alimentos para a complementação do leite materno. Várias 
são as causas que podem contribuir para o desmame precoce. As razões alegadas pelas mães para a não aderência ao AME, podem estar relacionadas à deficiência orgânica da mãe, problema com o bebê, atribuição de responsabilidade à mãe e a influência de terceiros. Dessa forma, não existem causas isoladas para o estabelecimento da amamentação, mas sim, a relação de fatores que existem entre a mãe e o bebê e o contexto em que estão inseridos (Bueno, Gomez, 2013).

Sendo assim, o desmame precoce pode interferir em inúmeros fatores de desenvolvimento do RN, tornando imprescindível a atuação do Fonoaudiólogo no atendimento desta população, levando orientações para as mães, identificando hábitos orais inadequados do bebê e aplicando terapia fonoaudiológica quando necessária (Oliveira et al., 2019). A partir do exposto, a presente pesquisa teve como objetivo verificar a relação entre aleitamento e e os reflexos na motricidade orofacial.

\section{Metodologia}

\section{Protocolo e registro}

A presente revisão que visa verificar o impacto do desmame em recém nascidos e os reflexos na motricidade orofacial, foi realizada conforme as recomendações PRISMA (Preferred Reporting Items for Systematic reviews and MetaAnalyses) (Moher et al., 2015). As buscas por artigos científicos foram conduzidas por dois pesquisadores independentes nas bases de dados eletrônicas MEDLINE (Pubmed), LILACS, SciELO, SCOPUS, WEB OF SCIENCE e BIREME, sem restrição de idioma e localização, durante o período de 2010 a 2020. A pesquisa foi estruturada e organizada na forma PICOS (Tabela 1), que representa um acrônimo para População alvo, Intervenção, Comparação, “Outcomes” (desfechos), Study. População de interesse ou problema de saúde $(\mathrm{P})$ corresponde a recém nascidos; intervenção (I): desmame; comparação (C): motricidade orofacial; outcome $(\mathrm{O})$ : impacto; $(\mathrm{S})$ : estudo transversal, estudo observacional, relatos de caso, estudos de casocontrole, ensaios clínicos controlados, estudos de coorte.

Tabela 1. Descrição dos componentes do PICOS.

\begin{tabular}{c|c}
\hline Acrônimo & Definição \\
\hline $\mathbf{P}$ & Recém nascidos \\
\hline $\mathbf{I}$ & Desmame \\
\hline $\mathbf{C}$ & Motricidade Orofacial \\
\hline $\mathbf{O}$ & Impacto \\
\hline $\mathbf{S}$ & Estudo transversal Estudo \\
& $\begin{array}{c}\text { Ebservacional Relatos de caso } \\
\text { Estudos de caso-controle } \\
\end{array}$ \\
& $\begin{array}{c}\text { Ensaios clínicos controlados } \\
\text { Estudos de coorte }\end{array}$ \\
\hline
\end{tabular}

Fonte: Autores.

\section{Estratégia de pesquisa}

Os descritores foram selecionados a partir dos vocabulários controlados Descritores em Ciências da Saúde (DeCS) e Medical Subject Heading Terms (MeSH), haja vista a sua grande utilização pela comunidade científica para a indexação de artigos na base de dados PubMed. Utilizou-se como estratégia de busca a combinação de descritores e operadores booleanos: (orofacial motricity) and (Breastfeeding) or (Weaning) and (development) and (newborn). A busca ocorreu de forma concentrada em maio de 2021. Para complementar e evitar viés de risco foi realizada uma busca por literatura cinza no Google Scholar. 
Critérios de Elegibilidade: Foram inclusos estudos sem restrição de idioma e localização, durante o período de 2010 a 2021. A Tabela 2 representa os critérios de inclusão e exclusão desenvolvidos nesta pesquisa. O estudo obteve pontuação 12 no protocolo modificado de Pithon et al. (2015) para avaliação da qualidade dos mesmos. Aqui não fecha com o que está no resumo.

Tabela 2. Síntese dos critérios de inclusão/exclusão.

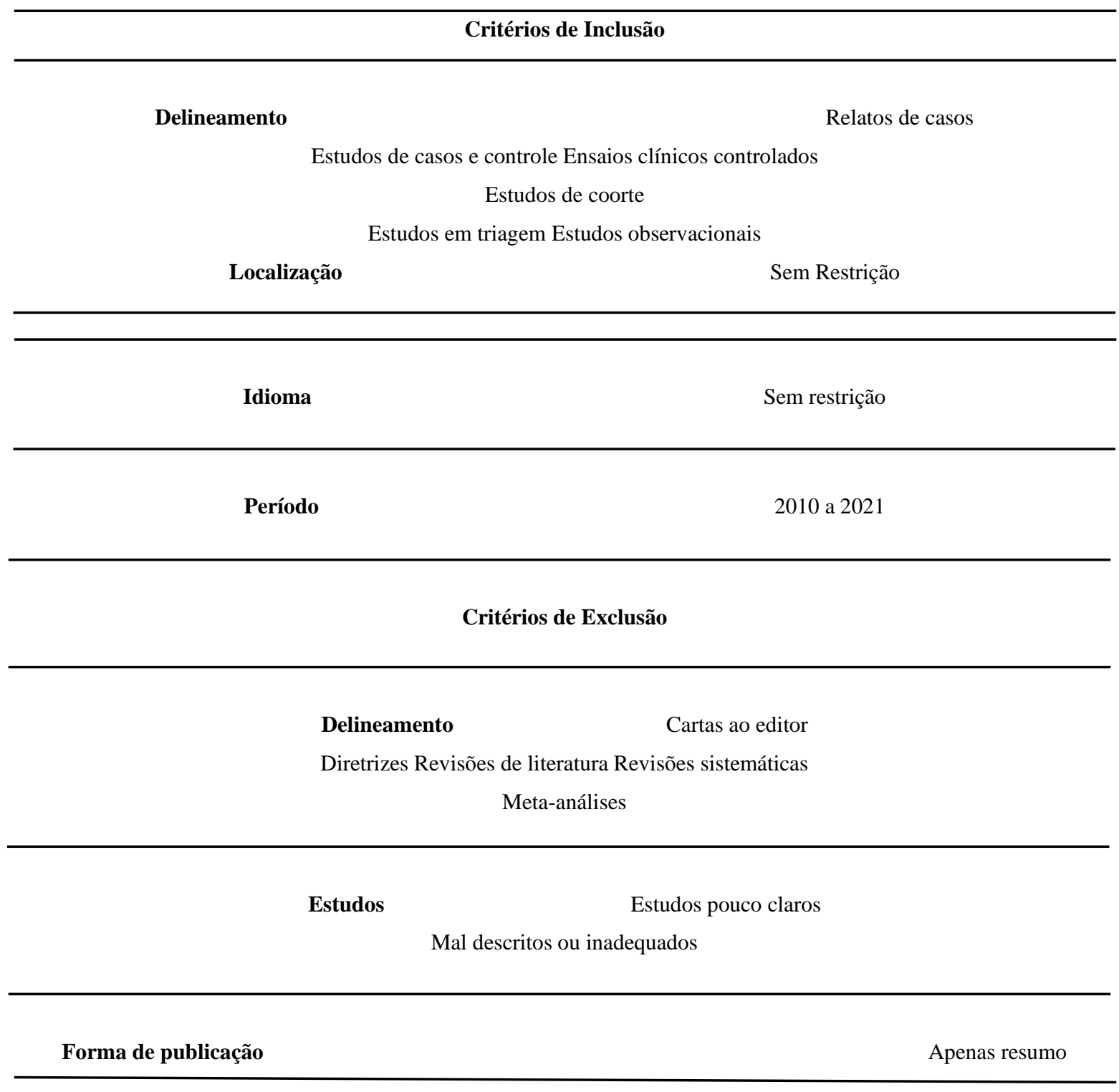

Fonte: Autores.

\section{Risco de viés}

A qualidade dos métodos utilizados no estudo incluído foi avaliada pelos revisores de forma independente (PH e APBD), de acordo com a recomendação PRISMA (Pithon et al., 2015). A avaliação priorizou a descrição clara das informações. Neste ponto, a revisão foi realizada às cegas, mascarando os nomes dos autores e revistas, evitando qualquer viés potencial e conflito de interesses.

\section{Análise dos dados}

A extração dos dados para o processo de elegibilidade dos estudos foi realizada utilizando-se uma ficha própria para 
revisão sistemática elaborada por dois pesquisadores em Programa Excel ${ }^{\circledR}$, na qual os dados extraídos foram adicionados por um dos pesquisadores e, então, conferidos por outro pesquisador. Inicialmente foram selecionados de acordo com o título; em seguida, os resumos foram analisados e apenas os que fossem potencialmente elegíveis foram selecionados. Com base nos resumos, artigos foram selecionados para leitura integral e foram admitidos os que atendiam a todos os critérios prédeterminados.

\section{Forma de seleção dos estudos}

Inicialmente os revisores de elegibilidade (GMBB e LFG) foram calibrados para a realização da revisão sistemática por APBD e PH. Após a calibração e esclarecimentos de dúvidas, os títulos e resumos foram examinados pelos revisores de elegibilidade (APBD e PH), de forma independente, os quais não estavam cegos para o nome dos autores e das revistas. Aqueles que apresentaram um título dentro do âmbito, mas os resumos não estavam disponíveis, também foram obtidos e analisados na íntegra. Posteriormente, os estudos elegíveis preliminarmente tiveram o texto completo obtido e avaliado.

\section{Dados Coletados}

Após a triagem, o texto do artigo selecionado foi revisado e extraído de forma padronizada por dois autores (GMBB e LFG) sob a supervisão de PH e APBD, identificando-se ano de publicação, local da pesquisa, idioma de publicação, tipo de estudo, amostra, método, resultado e conclusão do estudo.

\section{Resultado clínico}

O resultado clínico de interesse consistiu em verificar o impacto do desmame no recém nascido e os reflexos na motricidade orofacial. Aqueles que não utilizaram a abordagem definida não fizeram parte da amostra da revisão sistemática.

\section{Resultados}

Inicialmente a partir dos descritores eleitos, foram selecionados 75 artigos, dimensionado para 70 após exclusão por repetição; em seguida, os títulos e resumos foram analisados e 67 trabalhos foram excluídos, pois não estavam no escopo da proposta da pesquisa. Sendo então admitidos para a análise final três artigos no qual todos que foram inclusos respondiam a pergunta norteadora proposta (Figura 1). Os desenhos dos estudos selecionados foram do tipo transversal e coorte. 
Research, Society and Development, v. 10, n. 11, e499101120011, 2021

(CC BY 4.0) | ISSN 2525-3409 | DOI: http://dx.doi.org/10.33448/rsd-v10i11.20011

Figura 1. Diagrama de identificação e seleção de artigos adaptados das diretrizes Prisma.
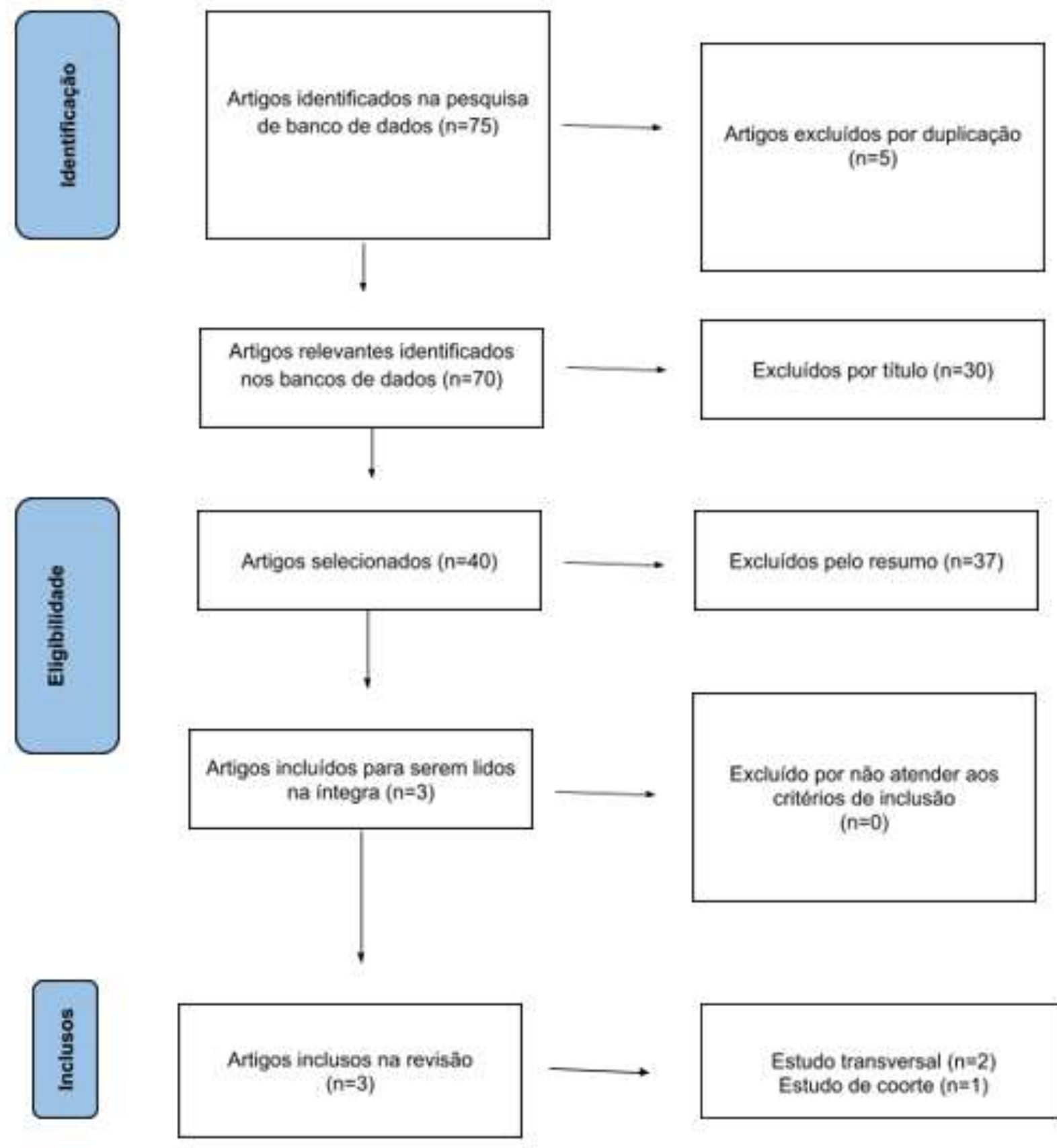

Estudo transversal $(n=2)$ Estudo de coorte (n=1)

Fonte: Autores. 
Tabela 3. Classificação das referências obtidas nas bases de dados Pubmed, Scielo, Lilacs, Web Of Science, Scopus e Bireme.

\begin{tabular}{|c|c|c|c|c|c|}
\hline Descritores & $\begin{array}{l}\mathbf{N}^{0} \text { total de } \\
\text { artigos }\end{array}$ & $\begin{array}{l}\mathrm{N}^{\circ} \text { de } \\
\text { Referências } \\
\text { excluídas }\end{array}$ & Motivo da exclusão & $\begin{array}{l}\mathrm{N}^{\circ} \mathrm{de} \\
\text { artigos } \\
\text { selecionados }\end{array}$ & $\begin{array}{l}\text { Banco de } \\
\text { dados }\end{array}$ \\
\hline \multirow{2}{*}{$\begin{array}{l}\text { (orofacial motricity) and } \\
\text { (Breastfeeding) or } \\
\text { (Weaning) and } \\
\text { (development) and } \\
\text { (newborn) }\end{array}$} & 75 & 72 & $\begin{array}{l}\text { Excluídos por título (30) } \\
\text { Excluídos por resumo (37) }\end{array}$ & 3 & PUBME \\
\hline & & & Artigos duplicados (5) & & \\
\hline $\begin{array}{l}\text { (orofacial motricity) and } \\
\text { (Breastfeeding) or } \\
\text { (Weaning) and } \\
\text { (development) and } \\
\text { (newborn) }\end{array}$ & 0 & 0 & 0 & 0 & $\begin{array}{l}\text { LILAC } \\
\mathrm{S}\end{array}$ \\
\hline $\begin{array}{l}\text { (orofacial motricity) and } \\
\text { (Breastfeeding) or } \\
\text { (Weaning) and } \\
\text { (development) } \\
\text { and (newborn) }\end{array}$ & 0 & 0 & 0 & 0 & $\begin{array}{l}\text { SCIEL } \\
\mathrm{O}\end{array}$ \\
\hline $\begin{array}{l}\text { (orofacial motricity) and } \\
\text { (Breastfeeding) or } \\
\text { (Weaning) and } \\
\text { (development) } \\
\text { and (newborn) }\end{array}$ & 0 & 0 & 0 & 0 & $\begin{array}{l}\text { SCOP } \\
\text { US }\end{array}$ \\
\hline $\begin{array}{l}\text { (orofacial motricity) and } \\
\text { (Breastfeeding) or } \\
\text { (Weaning) and } \\
\text { (development) } \\
\text { and (newborn) }\end{array}$ & 0 & 0 & 0 & 0 & $\begin{array}{l}\text { WEB } \\
\text { OF } \\
\text { SCIEN } \\
\text { CE }\end{array}$ \\
\hline $\begin{array}{l}\text { (orofacial motricity) and } \\
\text { (Breastfeeding) or } \\
\text { (Weaning) and } \\
\text { (development) } \\
\text { and (newborn) }\end{array}$ & 0 & 0 & 0 & 0 & $\begin{array}{l}\text { BIRE } \\
\text { ME }\end{array}$ \\
\hline Total & 75 & 72 & $\begin{array}{l}\text { Excluídos por título (30) } \\
\text { Excluídos por } \\
\text { resumo (36) } \\
\text { Artigos duplicados (5) }\end{array}$ & 3 & $\begin{array}{l}\text { PUBM } \\
\text { ED }\end{array}$ \\
\hline
\end{tabular}

Fonte: Autores.

\section{Desenho dos estudos}

O primeiro estudo (Pires, Giugliani, Silva, 2012) incluso nesta análise apresentava como objetivo investigar a associação entre a duração do aleitamento materno e a qualidade da função mastigatória em pré-escolares. A amostra foi composta por crianças acompanhadas desde o nascimento até as idades de três a cinco anos. Foram realizadas entrevistas com os responsáveis e coletados dados sobre hábitos alimentares e de sucção, presencialmente e por telefone, desde o nascimento até os 180 dias de vida das crianças. Quando atingiram três e cinco anos de idade, foi realizada avaliação mastigatória por fonoaudióloga em consultório particular (examinador desconhecia os hábitos alimentares e não nutritivos de sucção), fazendo a oferta de três alimentos de diferentes consistências. Cinco parâmetros da função mastigatória foram avaliados: incisão, competência labial, padrão mastigatório, movimentos mastigatórios e uso dos músculos periorais. Como cinco parâmetros foram avaliados durante a mastigação de três alimentos diferentes, os escores totais poderiam variar de zero (pior desempenho mastigatório) a 15 (melhor desempenho mastigatório). Todas as avaliações foram filmadas e as análises estatísticas foram 
realizadas no ambiente de software Statistical Package for the Social Sciences 13.0 (SPSS Inc., Chicago, IL, EUA).

O segundo estudo (Neu et al., 2013) teve como objetivo investigar a relação do tempo e tipo de aleitamento com as funções de mastigação, deglutição e respiração. A amostra foi constituída por participantes, de ambos os sexos, na faixa etária entre cinco e oito anos e nove meses de idade, de oito escolas públicas e particulares.

Foi aplicado um questionário para coletar dados de identificação das crianças, referentes ao tipo (peito exclusivo; mamadeira exclusiva; ou misto) e tempo de amamentação recebido (nenhum ou pouco - amamentada até 3 meses de idade; até 2 anos - recebeu aleitamento entre 4 meses e 2 anos de idade; e além de 2 anos - ofertado aleitamento além desta idade) e à respiração (presença de ronco e baba noturnos; permanência de lábios entreabertos/abertos durante o dia e presença de garganta seca ao acordar). Posteriormente os participantes foram submetidos à avaliação clínica, sendo avaliados individualmente pelo mesmo examinador, utilizando o Protocolo de Avaliação Miofuncional Orofacial com Escores Expandido -AMIOFE- E (FELÍCIO et al., 2010 ), adaptado para esta pesquisa. Foram coletados dados referentes às funções de mastigação, deglutição e respiração. Para avaliar a mastigação, solicitou-se que as crianças mastigassem três porções de pão francês de modo habitual, considerando o padrão mastigatório como normal ( mastigação bilateral alternada) ou alterado (mastigação bilateral simultânea, mastigação unilateral preferencial, mastigação unilateral crônica ou mastigação anterior) (NEU et al., 2013). A deglutição foi avaliada com água (beber de forma habitual) e pão francês, sendo que a presença de no mínimo três itens do protocolo definiu a deglutição como alterada. A respiração foi avaliada clinicamente por meio de observação durante conversa informal com as crianças e do teste da água. Considerou-se respiradora oral, quando a criança apresentou alteração em pelo menos 2 itens do protocolo: questionário, inspeção visual e teste da água.

Já a terceira pesquisa (SILVEIRA et al., 2013) teve como objetivo avaliar a influência de hábitos orais e do aleitamento materno sobre as habilidades orais de crianças. Foram selecionadas 125 crianças entre agosto de 2010 e março de 2011. Os critérios de inclusão consideraram crianças nascidas a termo, saudáveis, com idade entre oito meses e um dia e nove meses e 29 dias. E os de exclusão foram presença de alterações neurológicas, deformidades craniofaciais, síndrome genética e distúrbios sensoriais (visuais e auditivos).

Foi utilizado o Protocolo de avaliação das habilidades orais para crianças de oito a dez meses, o qual constituiu-se de 11 itens que avaliam as habilidades orais durante a sucção (ao seio ou mamadeira), com o uso do copo, com o uso da colher e durante a mastigação de alimento sólido. Para cada item atribuiu-se a pontuação 0,1 ou 2, de acordo com ausência, presença ocasional ou presença constante do item avaliado, respectivamente. A pontuação máxima possível para a sucção foi 6, para o uso do copo 8 , para o uso da colher 2, e para a mastigação 6 . Foi considerada habilidade oral adequada para a sucção o escore de 5-6, para o uso do copo 7-8, para o uso da colher 2, e para a mastigação 5-6. O instrumento foi aplicado por uma fonoaudióloga. $\mathrm{O}$ alimento para a criança foi ofertado por um familiar, utilizando seus próprios utensílios.

\section{Principais achados dos estudos}

As características principais das pesquisas selecionadas para este estudo, encontram-se expostas na Tabela 4. 
Tabela 4. Síntese dos artigos incluídos.

\begin{tabular}{|c|c|c|c|c|c|}
\hline $\begin{array}{l}\text { Autor/ Ano/ } \\
\text { Local de } \\
\text { publicação }\end{array}$ & Objetivo & $\mathbf{N}^{\circ}$ & Método & Resultados & Conclusão \\
\hline $\begin{array}{l}\text { Pires, } \\
\text { Giugliani, } \\
\text { Silva } \\
2012 \\
\text { Brasil }\end{array}$ & $\begin{array}{l}\text { Investigar a } \\
\text { associação entre a } \\
\text { duração do } \\
\text { aleitamento } \\
\text { materno e a } \\
\text { qualidade da } \\
\text { função } \\
\text { mastigatória em } \\
\text { pré-escolares }\end{array}$ & 144 & $\begin{array}{l}\text { Dados sobre parâmetros } \\
\text { sociodemográficos, dietéticos e } \\
\text { relacionados à sucção foram } \\
\text { coletados logo após o } \\
\text { nascimento e aos } 7,30,60,120 \\
\text { e } \\
180 \text { dias de vida. A função } \\
\text { mastigatória foi avaliada entre } \\
\text { as idades de } 3 \text { e } 5 \text { anos, por } \\
\text { meio de procedimento } \\
\text { padronizado envolvendo três } \\
\text { alimentos de diferentes } \\
\text { consistências. }\end{array}$ & $\begin{array}{l}\text { Foi encontrada correlação } \\
\text { positiva entre a duração da } \\
\text { amamentação e os escores da } \\
\text { função mastigatória. } \\
\text { Crianças amamentadas por } \\
\text { pelo menos } 12 \text { meses tiveram } \\
\text { escores significativamente } \\
\text { maiores, independentemente } \\
\text { do uso de mamadeira ou } \\
\text { chupeta. } \\
\text { Crianças que foram } \\
\text { amamentadas por mais tempo } \\
\text { tiveram maior probabilidade } \\
\text { de pontuar satisfatoriamente } \\
\text { em todos os parâmetros } \\
\text { testados. }\end{array}$ & $\begin{array}{l}\text { A amamentação } \\
\text { tem um impacto } \\
\text { positivo na } \\
\text { mastigação. A } \\
\text { duração do } \\
\text { aleitamento } \\
\text { materno associou- } \\
\text { se positivamente } \\
\text { à qualidade da } \\
\text { função } \\
\text { mastigatória na } \\
\text { idade pré-escolar. }\end{array}$ \\
\hline \multirow[t]{2}{*}{$\begin{array}{l}\text { Neu et } \\
\text { al., } \\
2013 \\
\text { Brasil }\end{array}$} & $\begin{array}{c}\text { investigar a } \\
\text { relação do tempo } \\
\text { e tipo de } \\
\text { aleitamento com } \\
\text { as funções de } \\
\text { mastigação, } \\
\text { deglu- tição e } \\
\text { respiração. }\end{array}$ & 52 & $\begin{array}{l}\text { Utilizando-se de questionário, } \\
\text { foram coletados dados } \\
\text { referentes ao tipo e tempo } \\
\text { de amamentação e respiração } \\
\text { das crianças. As mesmas }\end{array}$ & $\begin{array}{l}\text { Encontrou-se significância } \\
\text { estatística entre tipo de } \\
\text { aleitamento e a mastigação, } \\
\text { mas não entre o tempo de } \\
\text { aleitamento e esta variável. }\end{array}$ & $\begin{array}{l}\text { Houve relação } \\
\text { entre o tipo de } \\
\text { aleitamento e a } \\
\text { mastigação. }\end{array}$ \\
\hline & & & $\begin{array}{l}\text { foram submetidas à avaliação } \\
\text { clínica das funções, a qual } \\
\text { baseou-se no Protocolo de } \\
\text { Avaliação Miofuncional } \\
\text { Orofacial com Escores } \\
\text { Expandido } \\
\text { (AMIOFE- E). }\end{array}$ & & \\
\hline $\begin{array}{c}\text { Silveira et } \\
\text { al., } 2013 \\
\text { Brasil }\end{array}$ & $\begin{array}{c}\text { Avaliar a influência } \\
\text { de hábitos orais e } \\
\text { do aleitamento } \\
\text { materno sobre as } \\
\text { habilidades orais de } \\
\text { crianças }\end{array}$ & 125 & $\begin{array}{l}\text { As variáveis estudadas } \\
\text { incluíram avaliação das } \\
\text { habilidades orais e informações } \\
\text { sobre o aleitamento materno e a } \\
\text { introdução da alimentação } \\
\text { complementar. }\end{array}$ & $\begin{array}{l}\text { O aleitamento materno } \\
\text { influenciou positivamente a } \\
\text { aquisição das habilidades } \\
\text { orais de sucção das crianças } \\
\text { aos nove meses de idade. }\end{array}$ & $\begin{array}{l}\text { O aleitamento } \\
\text { materno } \\
\text { contribuiu para o } \\
\text { amadurecimento } \\
\text { orofacial, pois } \\
\text { melhorou a } \\
\text { habilidade oral } \\
\text { de sucção. }\end{array}$ \\
\hline
\end{tabular}

Fonte: Pires, Giugliani, Silva (2012); Neu et al., (2013); Silveira et al., (2013).

No primeiro estudo (Pires, Giugliani, Silva, 2012), foi encontrada correlação positiva entre a duração da amamentação e os escores da função mastigatória. Os escores médios da função mastigatória foram significativamente maiores entre as crianças amamentadas por 12 meses ou mais, alimentadas com mamadeira por no máximo 12 meses e que não usaram chupeta por mais de seis meses.

Ademais, os autores citam a associação significativa entre a manutenção da amamentação por 12 meses ou mais e a função mastigatória superior. Em média, crianças que foram amamentadas por 12 meses ou mais obtiveram dois pontos a mais na escala de função mastigatória, independentemente do uso de mamadeira ou da chupeta. Por outro lado, o uso desses dispositivos foi associado a uma redução de 3 ou 1,4 pontos nos escores da função mastigatória, respectivamente (Pires, Giugliani, Silva, 2012). A partir disso, crianças que foram amamentadas por mais tempo tiveram maior chance de escores 
satisfatórios em todos os cinco parâmetros e nos três diferentes alimentos testados, mais especificamente no padrão mastigatório e nos parâmetros de movimento mastigatório (Pires, Giugliani, Silva, 2012).

No segundo estudo (Neu et al., 2013), observaram-se dados descritivos referentes ao tipo de amamentação, tempo de aleitamento materno, tempo de aleitamento artificial, respiração, deglutição e mastigação. Verificou-se neste estudo que o tipo de aleitamento prevalente foi o misto, pois $61,54 \%$ das crianças foram amamentadas no peito e na mamadeira. Quanto ao tempo de aleitamento natural, observou-se que a maioria (40,38\% das crianças) não recebeu aleitamento, ou o recebeu por pouco tempo.

No que se refere ao tempo de aleitamento artificial, constatou-se que $71,15 \%$ das crianças estudadas receberam este tipo de aleitamento por mais de dois anos. Tanto a função de respiração, quanto de deglutição, apresentaram-se normais neste estudo. Observou-se que $75,00 \%$ das crianças eram respiradoras nasais e 67,30\% apresentaram deglutição normal. Quanto à mastigação, 53,85\% das crianças deste estudo, apresentaram esta função alterada, os autores descrevem que o tipo de aleitamento foi relacionado com a função mastigatória. Assim, verificou-se que, nesta pesquisa, os fatores tempo e tipo de amamentação não influenciaram no desenvolvimento de alterações, na maioria da amostra.

O terceiro estudo (Silveira et al., 2013) obteve como principal achado, Avaliar a influência de hábitos orais e do aleitamento materno sobre as habilidades orais de crianças. Sendo assim, foi observado em $89,7 \%$ das crianças em aleitamento materno uma habilidade oral de sucção adequada, e em 95,5\% das que não usavam chupeta, com diferença significativa em relação às que não eram amamentadas e usavam chupeta. A presença da amamentação aumentou 3,1 vezes a chance de a criança ter habilidade oral adequada para a sucção. A chupeta associou-se negativamente à habilidade de sucção, diminuindo a chance do desenvolvimento adequado desta idade. E o hábito de levar objetos à boca favoreceu as habilidades orais com o uso do copo.

\section{Discussão}

A amamentação serve como fonte de nutrição ideal para o desenvolvimento infantil, principalmente quando a nutrição materna é de qualidade. São inúmeras as vantagens que a amamentação oferece na sobrevivência das crianças através da obtenção dos nutrientes, tendo como exemplos os fatores de proteção imunológica, além de promover o crescimento e desenvolvimento adequado das estruturas orofaciais (Galvão, 2020). Além disso, o ato de amamentar auxilia no desenvolvimento dento facial, trazendo benefícios para a obtenção de uma oclusão normal e uma mastigação correta no futuro, tornando a face e a cavidade bucal um sistema equilibrado e dinâmico (Rondinel, 2018).

De acordo com Pires, Giugliani, Silva (2012), há um número maior de crianças do estudo que foram amamentadas por dois anos ou mais. Diante disso, crianças amamentadas por mais tempo tiveram maiores chances de escores satisfatórios, particularmente no padrão mastigatório e parâmetros de movimento da mastigação, concluindo os efeitos positivos que a amamentação promove na função mastigatória. Isto vem ao encontro com os achados de Rondinel (2018), o qual evidenciou a importância da amamentação exclusiva na promoção do adequado desenvolvimento do crânio e da face, devido à intensa atividade muscular necessária, que favorece a maturação dos músculos mastigatórios e desenvolvimento dos maxilares.

Verificou-se no estudo de Neu et al. (2013), que o tipo de aleitamento prevalente foi o misto, uma vez que a maioria das crianças foram amamentadas no peito e na mamadeira. Silva et al. (2019), avaliaram em seu estudo a influência do tipo de aleitamento materno e a relação com o consumo de vitaminas nos primeiros anos de vida e concluíram que, as crianças que estavam em aleitamento materno misto, artificial ou que não foram amamentadas até o $6^{\circ}$ mês de vida apresentaram menor consumo de vitamina A.

Quanto ao tempo de aleitamento materno, observou-se que 40,38\% das crianças não receberam aleitamento natural ou receberam por pouco tempo. Já Neu et al. (2013), constataram que a maioria das crianças estudadas receberam aleitamento 
artificial por mais de dois anos. O desmame precoce pode estar relacionado a vários fatores como: a mãe possuir trabalho informal ou estar desempregada, o grau de escolaridade, a idade materna, a alegação de pouco leite, o grau de aceitação e planejamento da gravidez, o vínculo afetivo com o parceiro e a influência da mãe nessa escolha (Sousa et al., 2015). A partir da constatação dos prejuízos advindos do desmame precoce, a Organização Mundial da Saúde e o Fundo das Nações Unidas firmaram parcerias entre os diversos países visando à redução nas taxas de mortalidade infantil. No Brasil, a primeira iniciativa aconteceu na década de 1980, com a implantação do Pr grama de Incentivo ao Aleitamento Materno (Brasil, 2010).

Em relação à função de respiração e deglutição, apresentaram-se normais no estudo de Neu et al. (2013) os quais observaram que a maior parte das crianças eram respiradoras nasais e mais da metade apresentaram deglutição normal. Assim, verificou-se que, nesta pesquisa, os fatores tempo e tipo de amamentação não influenciaram no desenvolvimento de alterações orofaciais. Entretanto, estes achados divergem dos encontrados na literatura, na qual Oliveira et al. (2015) analisaram a associação do período, tipo e forma de aleitamento com a ocorrência de hábitos orais deletérios e alterações de motricidade orofacial, constatando que quanto maior o tempo de aleitamento materno exclusivo, maior a proteção contra a ocorrência de hábitos e alterações no sistema estomatognático. Neiva et al. (2003), enfatizaram que o desmame precoce pode levar à ruptura do desenvolvimento motor-oral adequado, provocando alterações na postura e força dos OFAs, prejudicando as funções de mastigação, deglutição, respiração e articulação dos sons da fala.

Silveira et al. (2013), avaliaram a influência de hábitos orais e do aleitamento materno sobre as habilidades orais de crianças, sendo que dentre as crianças avaliadas, 96\% estavam com alimentação sólida na dieta e 54,4\% estavam em aleitamento materno exclusivo. Dessa forma, as habilidades orais adequadas para sucção foram observadas em $82,4 \%$ das crianças, enquanto o uso da chupeta influenciou negativamente tais habilidades. Isso corrobora Almeida et al. (2007), os quais relacionaram o desmame e a sucção de chupeta entre as crianças que apresentaram desmame precoce, observando que 47,6\% apresentavam o hábito de sucção de chupeta, e constataram que as crianças que obtiveram amamentação natural por mais de seis meses diminuíram a possibilidade de desenvolver hábitos orais deletérios, enquanto que as que foram desmamadas precocemente apresentaram maior probabilidade de adquiri-los. Ao relacionar o aleitamento materno com o sistema estomatognático, estudos evidenciam que o aleitamento materno favorece o crescimento e desenvolvimento orofacial existindo menor predisposição a desvios da normalidade (Oliveira et al.2015; Araújo et al. 2007).

\section{Considerações Finais}

De acordo com os dados obtidos, pode-se concluir que os benefícios que o aleitamento materno proporciona à saúde do bebê são indiscutíveis. Ao relacionar os achados desta pesquisa com o desmame precoce e seus reflexos no sistema estomatognático evidencia-se que o aleitamento materno natural oferece ao bebê um adequado desenvolvimento ósseo e muscular, garantindo, assim, o perfeito funcionamento das estruturas orais, possibilitando saúde geral à criança em virtude das inter relações existentes entre o sistema estomatognático e os demais órgãos e funções.

\section{Referências}

Alfaro Rondinel, E. J. (2018). Influencia de la lactancia materna en el desarrollo de maloclusiones en niños de 36 a 72 meses de edad en el colegio $6071-$ República Federal de Alemania de Villa el Salvador en el año 2018.

de Almeida, M. E. C., Melo, N. S., de Alencar Maia, S., \& da Costa, A. M. M. (2007). A influência do desmame precoce no desenvolvimento de hábitos bucais deletérios. ConScientiae Saúde, 6(2), 227-234.

de Araújo, C. M. T., da Silva, G. A. P., \& Coutinho, S. B. (2007). Aleitamento materno e uso de chupeta: repercussões na alimentação e no desenvolvimento do sistema sensório motor oral. Revista Paulista de Pediatria, 25(1), 59-65.

Bueno, K. D. C. V. N. (2013). A importância do aleitamento materno exclusivo até os seis meses de idade para a promoção de saúde da mãe e do bebê.

Benevenuto de Oliveira, M. M., Aparecida Silva, I., Bená Gregório, J. A., \& Pereira Gregório, E. (2015). Associação entre aleitamento materno, hábitos orais deletérios e alterações de motricidade orofacial em pacientes sob intervenção ortodôntica. Rev. iberoam. educ. invest. enferm.(Internet), 8-14. 
BRASIL. Ministério da Saúde, Secretaria de Atenção à Saúde, Departamento de Ações Programáticas e Estratégicas. Pesquisa de Prevalência de Aleitamento Materno em Municípios Brasileiros: situação do Aleitamento Materno em 227 municípios brasileiros. Brasília, 2010.

BRASIL. Ministério da Saúde. Secretaria de Atenção à Saúde. Departamento de Ações Programáticas e Estratégicas. II Pesquisa de prevalência de aleitamento materno nas capitais brasileiras e Distrito Federal. 1ª edição. Brasília, 2001.

BRASIL. Ministério da saúde: Caderneta da Gestante. Brasília- DF, 2014. www. Saúde.gov.br.

Delgado, S. E., \& Halpern, R. (2005). Amamentação de prematuros com menos de 1500 gramas: funcionamento motor-oral e apego. Pró-Fono Revista de Atualização Científica, 17, 141-152.

de Felício, C. M., Folha, G. A., Ferreira, C. L. P., \& Medeiros, A. P. M. (2010). Expanded protocol of orofacial myofunctional evaluation with scores: validity and reliability. International journal of pediatric otorhinolaryngology, 74(11), 1230-1239

Galvão, H. M. S. P. (2020). A influência do aleitamento materno no desenvolvimento do sistema estomatognático.

de Menezes, R. R., Coelho, A. S., \& Lobo, M. R. G. (2019). A importância da amamentação na formação de vínculos afetivos saudáveis entre mamãe/bebê. BIUS-Boletim Informativo Unimotrisaúde em Sociogerontologia, 12(5), 1-15.

Moher, D., Shamseer, L., Clarke, M., Ghersi, D., Liberati, A., Petticrew, M., ... \& Stewart, L. A. (2015). Preferred reporting items for systematic review and meta-analysis protocols (PRISMA-P) 2015 statement. Systematic reviews, 4(1), 1-9.

Neiva, F. C. B., Cattoni, D. M., Ramos, J. L. D. A., \& Issler, H. (2003). Desmame precoce: implicações para o desenvolvimento motor-oral. Jornal de Pediatria, 79(1), 7-12.

Neu, A. P., Silva, A. M. T. D., Mezzomo, C. L., Busanello-Stella, A. R., \& Moraes, A. B. D. (2013). Relação entre o tempo e o tipo de amamentação e as funções do sistema estomatognático. Revista CEFAC, 15, 420-426.

Oliveira, F. B. N. D., Fernandes, C. P., Gurgel, L. G., Fujinaga, C. I., \& Almeida, S. T. D. (2019). Breastfeeding assessment protocols and Speech Therapy: an integrative literature review. Revista CEFAC, 21.

Pithon, M. M., Sant'Anna, L. I. D. A., Baião, F. C. S., dos Santos, R. L., da Silva Coqueiro, R., \& Maia, L. C. (2015). Assessment of the effectiveness of mouthwashes in reducing cariogenic biofilm in orthodontic patients: a systematic review. Journal of dentistry, 43(3), $297-308$.

Pires, S. C., Giugliani, E. R. J., \& da Silva, F. C. (2012). Influence of the duration of breastfeeding on quality of muscle function during mastication in preschoolers: a cohort study. BMC Public Health, 12(1), 1-6.

Silva, M. A., Soares, M. M., Fonseca, P. C. D. A., Vieira, S. A., Carvalho, C. A., Amaral, R. M., \& Novaes, J. F. D. (2019). Relação entre os tipos de aleitamento materno e o consumo de vitamina A e ferro em crianças de 6 a 12 meses. Ciência \& Saúde Coletiva, 24, 4009-4018.

Silveira, L. M. D., Prade, L. S., Ruedell, A. M., Haeffner, L. S. B., \& Weinmann, A. R. M. (2013). Aleitamento materno e sua influência nas habilidades orais de crianças. Revista de Saúde Pública, 47, 37-43.

Scheeren, B., Mengue, A. P. M., Devincenzi, B. S., Barbosa, L. D. R., \& Gomes, E. (2012). Condições iniciais no aleitamento materno de recém-nascidos prematuros. Jornal da Sociedade Brasileira de Fonoaudiologia, 24, 199-204.

de Sousa, F., de Lima Claro, M., de Sousa, A. L., de Oliveira Lima, L. H., \& da Silva Santana, A. G. (2015). Avanços e desafios do aleitamento materno no Brasil: uma revisão integrativa. Revista Brasileira em Promoção da Saúde, 28(3), 434-442.

UNICEF. Manual e aleitamento materno. https://www.unicef.pt/docs/manual_aleitameno.pdf. 2021.

World Health Organization. (2001). Department of Nutrition for Health and Development. Department of Child and Adolescent Health and Development. The optimal duration of exclusive breastfeeding: report of an expert consultation. http://www.who.int/nutrition/ publications/optimal_duration_of_exc_bfeeding_ report_eng.pdf 